\title{
Correction: McCarthy, C.; et al. Developing Picornaviruses for Cancer Therapy. Cancers 2019, 11,685
}

\author{
Cormac McCarthy ${ }^{1}$, Nadishka Jayawardena ${ }^{1}$, Laura N. Burga ${ }^{1, *}$ and Mihnea Bostina ${ }^{1,2, * \mathbb{D}}$ \\ 1 Department of Microbiology and Immunology, University of Otago, Dunedin 9016, New Zealand; \\ mccco791@student.otago.ac.nz (C.M.); jaygi915@student.otago.ac.nz (N.J.) \\ 2 Otago Micro and Nano Imaging, University of Otago, Dunedin 9016, New Zealand \\ * Correspondence: laura.burga@otago.ac.nz (L.N.B.); mihnea.bostina@otago.ac.nz (M.B.)
}

Received: 20 January 2020; Accepted: 21 February 2020; Published: 28 February 2020

The authors wish to make the following corrections to this paper [1]:

On page 14, in paragraph two of Chapter 4, Echoviruses, the sentence: "The United States, much of the European Union and Japan found a lack of experimental evidence to approve Rigvir in cancer treatment [99]" should be deleted.

On page 15, line one, the sentence: "One such retrospective study showed a statistically significant increase in 3-year survival of melanoma patients when treated with Rigvir post-surgery than surgery alone or with other immunomodulators [97]", should be changed to: "One such retrospective study showed a statistically significant 4.39-6.57-fold decrease in mortality of melanoma patients when treated with Rigvir post-surgery than surgery alone [97]".

On page 15, line three, the sentence: "For best efficacy, Rigvir is injected intratumourally rather than intramuscularly, increasing 5-year survival between $29.9 \%$ and $19.5 \%$ [97]" should be deleted.

On page 15, paragraph two, line seven, the sentence "In the case of the stage IV histiocytic sarcoma patient, Rigvir was administered as part of a therapeutic cocktail including multisite radiation therapy, doxorubicin, cyclophosphamide and helixor P" should read: "In the case of the stage IV histiocytic sarcoma patient, Rigvir was administered for about 2 years followed by multisite radiation therapy, doxorubicin, cyclophosphamide and helixor P."

On page 15, paragraph three, line two, the sentence "One patient with a basal cell carcinoma, a diagnosis with a median expected survival of five months, was treated with Rigvir post-surgery [101]" should read: "One patient with melanoma unknown primary brain metastasis, a diagnosis with a median expected survival of five months, was treated with Rigvir post-surgery [101]."

The authors would like to apologize for any inconvenience caused to the readers by these changes.

\section{Reference}

1. McCarthy, C.; Jayawardena, N.; Burga, L.N.; Bostina, M. Developing Picornaviruses for Cancer Therapy. Cancers 2019, 11, 685. [CrossRef] [PubMed]

(C) 2020 by the authors. Licensee MDPI, Basel, Switzerland. This article is an open access article distributed under the terms and conditions of the Creative Commons Attribution (CC BY) license (http://creativecommons.org/licenses/by/4.0/). 